\title{
On Refinement of Generic State-based Software Components
}

\author{
Sun Meng ${ }^{1}$ and Luís S. Barbosa ${ }^{2}$ \\ 1 LMAM, School of Mathematical Science \\ Peking University, China \\ sunmeng@water.pku.edu.cn \\ 2 Department of Informatics \\ Minho University, Portugal \\ lsb@di. uminho.pt
}

\begin{abstract}
This paper characterizes refinement of state-based software components modelled as pointed coalgebras for some Set endofunctors. The proposed characterization is parametric on a specification of the underlying behaviour model introduced as a strong monad. This provides a basis to reason about (and transform) state-based software designs.
\end{abstract}

Keywords: components, refinement, coalgebraic models.

\section{Introduction}

Component-based software development $[15,16]$ emerged as a promising paradigm to deal with the ever increasing need for mastering complexity, software evolution and reuse. From object-orientation it retains the basic principle of encapsulation of data and code. The emphasis, however, is shifted from (class) inheritance to (object) composition to avoid interference between the former and encapsulation and, thus, paving the way to a development methodology based on third-party assembly of components. In [3,2], the authors proposed a coalgebraic characterization of software components as specifications of statebased modules, encapsulating a number of services through a public interface and providing limited access to an internal state space. Component persist and evolve in time, being able to interact with the environment during their overall computation. This piece of research has been driven by two key ideas: first, the 'black-box' characterization of software components favors an observational semantics; secondly, the proposed constructions should be generic in the sense that they should not depend on a particular notion of component behaviour. This led both to the adoption of coalgebra theory [14] to capture observational semantics and to the abstract characterization of possible behaviour models (e.g., partiality or (different degrees of) non-determinism) by strong monads acting as parameters in the resulting calculus.

Within this approach, briefly reviewed in section 2 , a set of component connectors have been identified and their properties established as bisimilarity equations with respect to a generic behaviour model. Actually, the corner stone of 
our 'components as coalgebras' approach is the use of coinduction to prove results, where $\sim$ is the appropriate bisimilarity relation, as a basis for reasoning and transforming component-based designs. This paper provides a basis to extend the approach toward the inequational side through the discussion of suitable notions of refinement.

In broad terms refinement can be defined as a transformation of an 'abstract' into a more 'concrete' design, entailing a notion of substitution, but not necessarily equivalence. There is, however, a diversity of ways of understanding both what substitution means, and what such a transformation should seek for. In data refinement, for example, after Hoare's landmark paper [8], the 'concrete' model is required to have enough redundancy to represent all the elements of the 'abstract' one. This is captured by the definition of a surjection from the former into the latter (the retrieve map). Also substitution is regarded as 'complete' in the sense that the (concrete) operations accept all the input values accepted by the corresponding abstract ones, and, for the same inputs, the results produced are also the same, up to the retrieve map. This means that, if models are specified, as they usually are in model-oriented design methods like $\operatorname{VDM}[10]$, in terms of pre and post-conditions, the former are weakened and the latter strengthened, under refinement. In object-orientation, on the other hand, substitution is expressed in terms of behaviour subtyping [11] capturing the idea that 'concrete' objects behave similarly to objects in the 'abstract' class. Finally, refinement in process algebras is usually discussed in terms of several 'observation' preorders (see, for example, [7]), most of them justifying transformations entailing reduction of nondeterminism.

In general, refinement correctness means that the usage of a system according to its 'abstract' description is still valid if it is actually built according to the 'concrete' one. What is commonly understood by being a valid usage is that the corresponding observable consequences are still the same, or, in a less strict sense, a subset thereof. The exact definition, however, depends on the underlying behaviour model, which, in our approach to component modelling, is taken as a specification parameter. Therefore, the main contribution of this paper is a semantic characterization of refinement for state-based components, parametric on a strong monad intended to capture components' behavioural models.

After a brief review of the component calculus, in section 2, the paper discusses two levels of component refinement: the interface level, concerned with what one may call plugging compatibility, in section 3, and the behavioural one in section 4, which introduces forward and backward morphisms as refinement 'witnesses', and section 5 which builds on them to propose a family of refinement preorders. Section 6 proves soundness of simulations to establish behavioural refinement. A few examples, along with some prospects for future work, are presented in section 7 . 


\section{Components as Coalgebras}

In $[3,2]$ software components and connectors have been characterised as dynamic systems with a public interface and a private, encapsulated state. A typical example is LBuff: a connector modelling a buffered channel which occasionally looses messages, as represented below:

$$
\begin{aligned}
& I=M+1 \\
& \\
& O=1+M
\end{aligned}
$$

The put and pick operations are regarded as 'buttons' or 'ports', whose signatures are grouped together in the diagram ( $M$ stands for a message parameter type, $\mathbf{1}$ for the nullary datatype and + for 'datatype sum'). One might capture LBuff dynamics by a function $a_{\text {LBuff }}: U \times I \longrightarrow \mathcal{P}(U \times O)$ where $U$ denotes the space state. This describes how LBuff reacts to input stimuli, produces output data (if any) and changes state. It can also be written in a curried form as $\bar{a}_{\text {LBuff }}: U \longrightarrow \mathcal{P}(U \times O)^{I}$ that is, as a coalgebra [14] of signature $U \longrightarrow \mathrm{T} U$ where functor $\mathrm{T}$ captures the transition 'shape':

$$
\mathrm{T}=\mathcal{P}(\mathrm{ld} \times O)^{I}
$$

Built in this 'shape' is the possibility of non deterministic evolution captured by the use of $\mathcal{P}$, the finite powerset monad. Concretely, LBuff is defined over $U=M^{*}$, with nil as the initial state, and dynamics given by

$$
\begin{aligned}
a_{\text {LBuff }}\langle u, \text { put } m\rangle & =\left\{\left\langle u, \iota_{1} *\right\rangle,\left\langle m: u, \iota_{1} *\right\rangle\right\} \\
a_{\text {LBuff }}\langle u, \text { pick }\rangle & =\left\{\left\langle\text { tail } u, \iota_{2}(\text { head } u)\right\rangle\right\}
\end{aligned}
$$

where put $m$ and pick abbreviates $\iota_{1} m$ and $\iota_{2} *$, respectively.

Non determinism, capturing the occasional loss of messages, is a possible behavioural pattern for this buffer, but, by no means, the only one. Other components will exhibit different behaviour models: actually genericity is achieved by replacing the powerset monad above, by an arbitrary strong monad ${ }^{3} \mathrm{~B}$. In the general case, a component $p: I \longrightarrow O$ is specified as a (pointed) coalgebra in Set

$$
\left\langle u_{p} \in U_{p}, \bar{a}_{p}: U_{p} \longrightarrow \mathrm{B}\left(U_{p} \times O\right)^{I}\right\rangle
$$

\footnotetext{
${ }^{3} \mathrm{~A}$ strong monad is a monad $\langle\mathrm{B}, \eta, \mu\rangle$ where $\mathrm{B}$ is a strong functor and both $\eta$ and $\mu$ strong natural transformations. $\mathrm{B}$ being strong means there exist natural transformations $\tau_{r}^{\top}: \mathrm{T} \times-\Longrightarrow \mathrm{T}(\mathbf{I d} \times-)$ and $\tau_{l}^{\top}:-\times \mathrm{T} \Longrightarrow \mathrm{T}(-\times \mathbf{I d})$ called the right and left strength, respectively, subject to certain conditions. Their effect is to distribute the free variable values in the context "-" along functor B.
} 
where point $u_{p}$ is taken as the 'initial' or 'seed' state. Therefore, the computation of an action will not simply produce an output and a continuation state, but a B-structure of such pairs. The monadic structure provides tools to handle such computations. Unit $(\eta)$ and multiplication $(\mu)$, provide, respectively, a value embedding and a 'flatten' operation to reduce nested behavioural annotations. Strength, either in its right $\left(\tau_{r}\right)$ or left $\left(\tau_{l}\right)$ version, will cater for context information.

In such a framework, components become arrows in a (bicategorical) universe $\mathrm{Cp}$ whose objects are sets, which provide types to input/output parameters (the components' interfaces), and component morphisms $h: p \longrightarrow q$ are functions relating the state spaces of $p$ and $q$ and satisfying the following seed preservation and coalgebra conditions:

$$
h u_{p}=u_{q} \quad \text { and } \quad \bar{a}_{q} \cdot h=\mathrm{B}(h \times O)^{I} \cdot \bar{a}_{p}
$$

For each triple of objects $\langle I, K, O\rangle$, a composition law is given by functor $;_{I, K, O}$ : $\mathrm{Cp}(I, K) \times \mathrm{Cp}(K, O) \longrightarrow \mathrm{Cp}(I, O)$ whose action on objects $p$ and $q$ is

$$
\begin{gathered}
p ; q=\left\langle\left\langle u_{p}, u_{q}\right\rangle \in U_{p} \times U_{q}, \bar{a}_{p ; q}\right\rangle \quad \text { with } \\
a_{p ; q}=U_{p} \times U_{q} \times I \stackrel{\cong}{\longrightarrow} U_{p} \times I \times U_{q} \stackrel{a_{p} \times \mathrm{id}}{\longrightarrow} \mathrm{B}\left(U_{p} \times K\right) \times U_{q} \\
\stackrel{\tau_{r}}{\longrightarrow} \mathrm{B}\left(U_{p} \times K \times U_{q}\right) \stackrel{\mathrm{B}}{\longrightarrow} \mathrm{B}\left(U_{p} \times\left(U_{q} \times K\right)\right) \\
\stackrel{\mathrm{B}\left(\mathrm{id} \times a_{q}\right)}{\longrightarrow} \mathrm{B}\left(U_{p} \times \mathrm{B}\left(U_{q} \times O\right)\right) \stackrel{\mathrm{B} \tau_{l}}{\longrightarrow} \mathrm{BB}\left(U_{p} \times\left(U_{q} \times O\right)\right) \\
\stackrel{\mathrm{B}}{\longrightarrow}\left(U_{p} \times U_{q} \times O\right) \stackrel{\mu}{\longrightarrow} \mathrm{B}\left(U_{p} \times U_{q} \times O\right)
\end{gathered}
$$

Similarly, for each object $K$, an identity law is given by a functor $\operatorname{copy}_{K}: \mathbf{1} \longrightarrow$ $\mathrm{Cp}(K, K)$ whose action is the constant component $\left\langle * \in \mathbf{1}, \eta_{\mathbf{1} \times K}\right\rangle$. Note that the definitions above rely solely on the monadic structure of B.

In $[3,2]$ a set of component combinators have been defined upon $\mathrm{Cp}$ in a similar parametric way and their properties studied. In particular it was shown that any function $f: A \longrightarrow B$ can be lifted to $C p$ as $\ulcorner f\urcorner=\left\langle * \in \mathbf{1}, \eta_{(\mathbf{1} \times B)} \cdot\right.$ (id $\times$ $f)\rangle$. Also defined were both a wrapping mechanism $p[f, g]$ which encodes the preand post-composition of a component with $\mathrm{Cp}$-lifted functions, and three tensors, capturing, respectively, external choice $(\boxplus: I+J \longrightarrow O+R)$, parallel $(\otimes$ : $I \times J \longrightarrow O \times R$ ) and concurrent (困 : $I+J+I \times J \longrightarrow O+R+O \times R$ ) composition, given components $p: I \longrightarrow O$ and $q: J \longrightarrow R$. When interacting with $p \boxplus q$ : $I+J \longrightarrow O+R$, the environment chooses either to input a value of type $I$ or one of type $J$, which triggers the corresponding component ( $p$ or $q$, respectively), producing the relevant output. In its turn, parallel composition corresponds to a synchronous product: both components are executed simultaneously when triggered by a pair of legal input values. Note, however, that the behaviour effect, captured by monad B, propagates. For example, if B can express component failure and one of the arguments fails, the product will fail as well. Finally, concurrent composition combines choice and parallel, in the sense that $p$ and $q$ can be executed independently or jointly, depending on input. Generalized interaction is catered through a 'feedback' mechanism on a subset of the inputs. 


\section{Interface Refinement}

Component interface refinement is concerned with type compatibility. The question is whether a component can be transformed, by suitable wiring, to replace another component with a different interface. As the structure of components interface types encodes the available operations, this may capture situations of extension of functionality, in the sense that the 'concrete' component may introduce new operations. In the context of object-orientation, this is often called design sophistication (rather than refinement) and it is known not to be a congruence with respect to typical process combinators (see e.g., [17]). If we structure component input and output parameters as an operations' signature, interface refinement can also be seen as induced by a signature morphism, as in e.g., [13].

To motivate our own approach, consider, from [3], the following law expressing commutativity of choice:

$$
p \boxplus q \sim(q \boxplus p)\left[\mathbf{s}_{+}, \mathbf{s}_{+}\right]
$$

where $\mathrm{s}_{+}: I+J \longrightarrow J+I$ is a natural isomorphism capturing + commutativity. The law states that $p \boxplus q$ and $q \boxplus p$ are bisimilar up to isomorphic wiring. This means that the observational effect of component $p \boxplus q$ can be achieved by $q \boxplus p$, providing the interface of the latter is converted to the interface of the former. Such a conversion is achieved by composition with the appropriate wires, leading to a notion of replaceability.

Definition 1 Let $p$ and $q$ be components. We say that $p: I \longrightarrow O$ is replaceable by $q: I^{\prime} \longrightarrow O^{\prime}$, or $q$ is a replacement of $p$, and write $p \lessdot q$ if there exist functions $w_{1}: I \longrightarrow I^{\prime}$ and $w_{2}: O^{\prime} \longrightarrow O$, to be referred to as the replacement witnesses, such that

$$
p \sim q\left[w_{1}, w_{2}\right]
$$

Furthermore, components $p$ and $q$ are interchangeable if each of them is a replacement of the other. Formally,

$$
p \doteqdot q \quad \text { iff } \quad p \lessdot q \wedge q \lessdot p
$$

Clearly, $p \boxplus q \doteqdot q \boxplus p$, using isomorphism $\mathrm{s}_{+}$as a wire in both cases. In general, $p \doteqdot q$ whenever $w_{1}$ and $w_{2}$ in (5) are isomorphisms.

Lemma 1. Replaceability (๕) is a preorder on components

Proof. Clearly, $\lessdot$ is reflexive because $p \lessdot p$ is witnessed by $p \sim p$ [id, id]. On the other hand, if $p \lessdot q$ and $q \lessdot r$ hold, there exist $w_{1}, w_{2}, w_{3}$ and $w_{4}$ such that $p \sim q\left[w_{1}, w_{2}\right]$ and $q \sim r\left[w_{3}, w_{4}\right]$. Thus, a composition result on wrapping [2] and transitivity of $\sim$, entails $p \sim r\left[w_{1} \cdot w_{3}, w_{4} \cdot w_{2}\right]$, i.e., $p \lessdot r$. 
Using $\lessdot$ and $\doteqdot$, some component laws in [2], as (4) above, can be presented in a 'wiring free' form. As another example consider the law relating concurrent composition with choice,

$$
\left\ulcorner\iota_{1}\right\urcorner ;(p \boxplus q) \sim(p \boxplus q) ;\left\ulcorner\iota_{1}\right\urcorner
$$

which gives rise to two replacement inequations:

$$
\left\ulcorner\iota_{1}\right\urcorner ;(p \boxplus q) \lessdot p \boxplus q \quad \text { and } \quad(p \boxplus q) ;\left\ulcorner\iota_{1}\right\urcorner \lessdot p \boxplus q
$$

Finally, the statement that every component $p$ can replace an inert component can be expressed as an interface refinement situation: inert $\lessdot p$.

Relation $\lessdot$, however, fails to be a pre-congruence with respect to the component operators introduced in [3]. It is easy to check that $\boxplus, \otimes$ and $⿴$, as well as wrapping are preserved, i.e., if $p \lessdot p^{\prime}$ then, for any $q, f$ and $g, p[f, g] \lessdot p^{\prime}[f, g]$, $p \boxplus q \lessdot p^{\prime} \boxplus q$ and, similarly, for the other two tensors. But things are different with respect to sequential composition and feedback. In these cases, the replaced expression may even become wrongly typed.

What $p \lessdot p^{\prime}$ means is that component $p$ can be replaced in any context by $p^{\prime}\left[w_{1}, w_{2}\right]$, for any functions $w_{1}, w_{2}$ witnessing the fact. The explicit reference to them is actually required, something which is not completely satisfactory in a refinement situation, although common in similar settings (cf. [17]).

\section{Forward and Backward Morphisms}

Interface refinement is essentially concerned with plugging adjustment. Behaviour refinement, on the other hand, affects the internal dynamics of a component while leaving unchanged its external interface: it takes place inside each hom-category of $\mathrm{Cp}$. Intuitively component $p$ is a behavioural refinement of $q$ if the behaviour patterns observed from $p$ are a structural restriction, with respect to the behavioural model captured by monad B, of those of $q$. To make precise such a 'definition' we shall first describe behaviour patterns concretely as generalized transitions.

Actually, just as transition systems can be coded back as coalgebras, any coalgebra $\langle U, p: U \longrightarrow \mathrm{T} U\rangle$ specifies a (T-shaped) transition structure over its carrier $U$. For extended polynomial Set endofunctors ${ }^{4}$ such a structure may be expressed as a binary relation $\longrightarrow p \subseteq U \times U$, defined in terms of the structural membership relation $\in_{\mathrm{T}} \subseteq U \times \mathrm{T} U$, i.e.,

$$
u \longrightarrow p u^{\prime} \quad \text { iff } \quad u^{\prime} \in_{\mathrm{T}} p u
$$

\footnotetext{
4 The class inductively defined as the least collection of functors containing the identity Id and constant functors $\underline{K}$ for all objects $K$ in the category, closed by functor composition and finite application of product, coproduct, covariant exponential and finite powerset functors.
} 
where $\epsilon_{\mathrm{T}}$ is defined by induction of the structure of $\mathrm{T}$ :

$$
\begin{aligned}
& x \in_{\mathrm{Id}} y \text { iff } \quad x=y \\
& x \in_{\underline{K}} y \text { iff false } \\
& x \in_{\mathrm{T}_{1} \times \mathrm{T}_{2}} y \quad \text { iff } \quad x \in_{\mathrm{T}_{1}} \pi_{1} y \vee x \in \in_{\mathrm{T}_{2}} \pi_{2} y \\
& x \in \in_{\mathrm{T}_{1}+\mathrm{T}_{2}} y \quad \text { iff } \quad \begin{cases}y=\iota_{1} y^{\prime} & \Rightarrow x \in_{\mathrm{T}_{1}} y^{\prime} \\
y=\iota_{2} y^{\prime} & \Rightarrow x \in_{\mathrm{T}_{2}} y^{\prime}\end{cases} \\
& x \in_{\mathrm{T} \underline{K}} y \quad \text { iff } \quad \exists_{k \in K} \cdot x \in_{\mathrm{T}} y k \\
& x \in_{\mathcal{P} \mathrm{T}} y \quad \text { iff } \quad \exists_{y^{\prime} \in y} . x \in_{\mathrm{T}} y^{\prime}
\end{aligned}
$$

Notice that, given $x \in U, X \in \mathrm{T} U$ and a function $h: U \longrightarrow V$, if $x \in_{\mathrm{T}} X$ then $h x \in_{\mathrm{T}} \mathrm{T} h X$, as it may be shown by induction on the polynomial structure, resorting to the definition of $\epsilon_{\mathrm{T}}$ and functoriality. Similarly, the dynamics of $p: I \longrightarrow O$, based on functor $\mathrm{B}(\mathrm{Id} \times O)^{I}$, can be expressed in terms of the following transition relation:

$$
u \stackrel{\langle i, o\rangle}{\longrightarrow} u^{\prime} \quad \text { iff } \quad\left\langle u^{\prime}, o\right\rangle \in \mathrm{B}(p u) i
$$

In this setting, a possible (and intuitive) way of regarding component $p$ as a behavioural refinement of $q$ is to consider that $p$ transitions are simply preserved in $q$. For non deterministic components this is understood simply as set inclusion. But one may also want to consider additional restrictions. For example, to stipulate that if $p$ has no transitions from a given state, $q$ should also have no transitions from the corresponding state(s). Or one may adopt the dual point of view requiring transition reflection instead of preservation. In any case the same basic question arises: how can such a refinement stituation be identified?

In data refinement, as mentioned above, there is a 'recipe' to identify a refinement situation: look for a retrieve function to witness it. I.e., a morphism in the relevant category, from the 'concrete' to the 'abstract' model such that the latter can be recovered from the former up to a suitable notion of equivalence, though, typically, not in a unique way.

In our components' framework, however, things do not work this way. The reason is obvious: component morphisms are (seed preserving) coalgebra morphisms which are known (e.g., [14]) to entail bisimilarity. Therefore we have to look for a somewhat weaker notion of a morphism between coalgebras.

First of all recall that a component morphism from $p$ to $q$ is a seed preserving function $h: U_{p} \longrightarrow U_{q}$ such that

$$
\mathrm{B}(h \times \mathrm{id}) \cdot a_{p}=a_{q} \cdot(h \times i d)
$$

In terms of transitions, equation (7) is translated into the following two requirements (by a straightforward generalization of an argument in [14]):

$$
\begin{aligned}
u \stackrel{\langle i, o\rangle}{\longrightarrow} u^{\prime} & \Rightarrow h u \stackrel{\langle i, o\rangle}{\longrightarrow} h u^{\prime} \\
h u \stackrel{\langle i, o\rangle}{\longrightarrow} v^{\prime} & \Rightarrow \exists_{u^{\prime} \in U} \cdot u \stackrel{\langle i, o\rangle}{\longrightarrow} p u^{\prime} \wedge v^{\prime}=h u^{\prime}
\end{aligned}
$$


which jointly state that, not only $p$ dynamics, as represented by the induced transition relation, is preserved by $h(8)$, but also $q$ dynamics is reflected back over the same $h$ (9). Is it possible to weaken the morphism definition to capture only one of these aspects? The answer is given as follows:

An order $\leq$ on a Set endofunctor $\mathrm{T}$ is defined in [9] as a functor $\leq$ which makes the following diagram to commute:

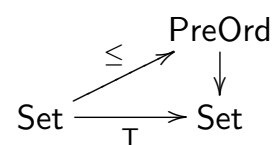

concretely

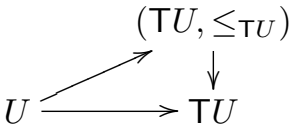

This means that for any function $h: X \longrightarrow Y$, Th preserves the order, i.e.

$$
x_{1} \leq_{\mathrm{T} X} x_{2} \Rightarrow(\mathrm{T} h) x_{1} \leq_{\mathrm{T} Y}(\mathrm{~T} h) x_{2}
$$

In the sequel $\leq$ will be referred to as a refinement preorder. Then,

Definition 2 Let $\mathrm{T}$ be an extended polynomial functor on Set and consider two $\mathrm{T}$-coalgebras $\alpha: U \longrightarrow \mathrm{T} U$ and $\beta: V \longrightarrow \mathrm{T} V$. A forward morphism $h: \alpha \longrightarrow \beta$ with respect to a refinement preorder $\leq$, is a function from $U$ to $V$ such that

$$
\mathrm{T} h \cdot \alpha \leq \beta \cdot h
$$

Dually, $h$ is called a backwards morphism if

$$
\beta \cdot h \leq \mathrm{T} h \cdot \alpha
$$

The following lemma shows that such morphisms compose and can be taken as witnesses of refinement situations:

Lemma 2. For $\mathrm{T}$ an endofunctor in Set, T-coalgebras and forward (respectively, backward) morphisms define a category.

Proof. In both cases, identities are the identities on the carrier and composition is inherited from Set. What remains to be shown is that the composition of forward (respectively, backward) morphisms yields again a forward (respectively, backward) morphism. So, let $h: \alpha \longrightarrow \beta$ and $k: \beta \longrightarrow \gamma$ be two forward (respectively, backward) morphisms. Then 
(forward case)

$$
\begin{aligned}
& \mathrm{T}(k \cdot h) \cdot \alpha \\
= & \quad\{\mathrm{T} \text { functor }\} \\
& \mathrm{T} k \cdot(\mathrm{T} h \cdot \alpha) \\
\leq & \quad\{h \text { forward and }(10)\} \\
& \mathrm{T} k \cdot(\beta \cdot h) \\
= & \quad\{\cdot \text { associative }\} \\
& (\mathrm{T} k \cdot \beta) \cdot h \\
\leq & \quad\{k \text { forward }\} \\
& (\gamma \cdot k) \cdot h \\
= & \quad\{\cdot \text { associative }\} \\
& \gamma \cdot(k \cdot h)
\end{aligned}
$$

(backward case)

$$
\begin{aligned}
& \gamma \cdot(k \cdot h) \\
= & \quad\{\cdot \text { associative }\} \\
& (\gamma \cdot k) \cdot h \\
\leq & \quad\{k \text { backward }\} \\
& (\mathrm{T} k \cdot \beta) \cdot h \\
= & \quad\{\cdot \text { associative }\} \\
& \mathrm{T} k \cdot(\beta \cdot h) \\
\leq & \quad\{h \text { backward and }(10)\}
\end{aligned}
$$$$
\mathrm{T} k \cdot \mathrm{T} h \cdot \alpha
$$$$
=\{\mathrm{T} \text { functor }\}
$$$$
\mathrm{T}(k \cdot h) \cdot \alpha
$$

Such a split of a coalgebra morphism, witnessing a bisimulation equation, into two conditions, makes it possible to capture separately transition preservation and reflection. To prove the next result, however, it is necessary to impose an extra condition on the refinement preorder $\leq$ expressing its compatibility with $\in_{\mathrm{T}}$ : for all $x \in X$ and $x_{1}, x_{2} \in \mathrm{T} X$,

$$
x \in_{\mathrm{T}} x_{1} \wedge x_{1} \leq x_{2} \Rightarrow x \in_{\mathrm{T}} x_{2}
$$

Lemma 3. Let $\mathrm{T}$ be an extended polynomial functor in Set, and $\alpha$ and $\beta$ two $\mathrm{T}$-coalgebras as above. Let $\longrightarrow_{\alpha}$ and $\longrightarrow_{\beta}$ denote the corresponding transition relations. A forward (respectively, backward) morphism $h: \alpha \longrightarrow \beta$ preserves (respectively, reflects) such transition relations.

Proof. Preservation follows from

$$
\begin{aligned}
& u \longrightarrow \alpha u^{\prime} \\
& \equiv \quad\{\longrightarrow \text { definition }\} \\
& u^{\prime} \in_{\mathrm{T}} \alpha u \\
& \Rightarrow \quad\left\{\in_{\mathrm{T}} \text { definition }\right\} \\
& h u^{\prime} \in_{\mathrm{T}}(\mathrm{T} h \cdot \alpha) u \\
& \equiv \quad\{h \text { forward and (11) }\} \\
& h u^{\prime} \in_{\mathbf{T}}(\beta \cdot h) u \\
& \equiv \quad\{\cdot \text { associative and } \longrightarrow \text { definition }\} \\
& h u \longrightarrow \beta h u^{\prime}
\end{aligned}
$$


To establish reflection suppose that $h u \longrightarrow \beta v^{\prime}$, i.e., $v^{\prime} \in_{\mathrm{T}}(\beta \cdot h) u$. As $h$ is a backward morphism we have $\beta \cdot h \leq \mathrm{T} h \cdot \alpha$, which, together with requirement (11), entails $v^{\prime} \in \in_{\mathrm{T}}(\mathrm{T} h \cdot \alpha) u$. This implies the existence of a state $u^{\prime} \in U$ such that $v^{\prime}=h u^{\prime}$ and $u^{\prime} \in_{\mathrm{T}} \alpha u$, i.e., $u \longrightarrow_{\alpha} u^{\prime}$.

\section{Behaviour Refinement}

The existence of a forward (backward) morphism connecting two components $p$ and $q$ witnesses a refinement situation whose symmetric closure coincides, as expected, with bisimulation. In the sequel we will restrict ourselves to forward refinement ${ }^{5}$ and define behaviour refinement as the existence of a forward morphism up to bisimulation. Formally,

Definition 3 Component $p$ is a behaviour refinement of $q$, written $q \unlhd p$, if there exist components $r$ and $s$ such that $p \sim r, q \sim s$ and a (seed preserving) forward morphism from $r$ to $s$.

The exact meaning of a refinement assertion $q \unlhd p$ depends, of course, on the concrete refinement preorder $\leq$ adopted. Let us consider a few possibilities.

- T-structural inclusion, defined by $x \leq y$ iff $\forall_{e \in_{\mathrm{T} x}} . e \in_{\mathrm{T}} y$, seems inadequate because the transition relation preserved by a forward morphism is not $\stackrel{\langle i, o\rangle}{\longrightarrow}$, but simply $\longrightarrow p$, and, therefore, blind to the outputs produced. This suggests an additional requirement on refinement preorders for $\mathrm{Cp}$ components: their definition on a constant functor $\underline{K}$ is equality on set $K$, i.e., $x \leq_{K} y$ iff $x={ }_{K} y$ so that transitions with different $O$-labels could not be related.

- Building on this idea, we arrive to a first (good) example:

$$
\begin{aligned}
& x \subseteq_{\text {Id }} y \text { iff } \quad x=y \\
& x \subseteq_{K} y \text { iff } \quad x={ }_{K} y \\
& x \subseteq \mathrm{T}_{1} \times \mathrm{T}_{2} y \quad \text { iff } \quad \pi_{1} x \subseteq \mathrm{T}_{1} \pi_{1} y \wedge \pi_{2} x \subseteq_{\mathrm{T}_{2}} \pi_{2} y \\
& x \subseteq_{\mathrm{T}_{1}+\mathrm{T}_{2}} y \quad \text { iff } \quad \begin{cases}x=\iota_{1} x^{\prime} \wedge y=\iota_{1} y^{\prime} & \Rightarrow x^{\prime} \subseteq_{\mathrm{T}_{1}} y^{\prime} \\
x=\iota_{2} x^{\prime} \wedge y=\iota_{2} y^{\prime} & \Rightarrow x^{\prime} \subseteq_{\mathrm{T}_{2}} y^{\prime}\end{cases} \\
& x \subseteq_{\mathrm{T} \underline{K}} y \quad \text { iff } \quad \forall_{k \in K} \cdot x k \subseteq_{\mathrm{T}} y k \\
& x \subseteq_{\mathcal{P} \mathrm{T}} y \quad \text { iff } \quad \forall_{e \in x} \exists_{e^{\prime} \in y} . e \subseteq_{\mathrm{T}} e^{\prime}
\end{aligned}
$$

A forward refinement of non deterministic components based on $\subseteq_{\mathrm{T}}$ captures the classical notion of nondeterminism reduction.

\footnotetext{
${ }^{5}$ A similar study can be made about backward refinement, although the underlying intuition seems less familiar.
} 
- However, this preorder can be tuned to more specific cases. For example, the following 'failure forcing' variant $-\subseteq_{\mathrm{T}}^{E}$, where $E$ stands for 'emptyset' - guarantees that the concrete component fails no more than the abstract one. It is defined as $\subseteq_{\mathrm{T}}$ by replacing the clause for the powerset functor by

$$
x \subseteq_{\mathcal{P} \mathrm{T}}^{E} y \quad \text { iff } \quad(x=\emptyset \Rightarrow y=\emptyset) \wedge \forall_{e \in x} \exists_{e^{\prime} \in y} . e \subseteq_{\mathrm{T}} e^{\prime}
$$

- Relation $\subseteq_{\mathrm{T}}$ is inadequate for partial components: refinement would collapse into bisimilarity, instead of entailing increasing definition in the implementation. An alternative is relation $\subseteq$ T ( $F$ standing for 'failure') which replaces the sum clause in $\subseteq$ T by

$$
x \subseteq_{\mathrm{T}_{1}+\mathrm{T}_{2}} y \text { iff } \begin{cases}x=\iota_{1} x^{\prime} \wedge y=\iota_{1} y^{\prime} & \Rightarrow x^{\prime} \subseteq \mathrm{T} y^{\prime} \\ x=\iota_{2} * & \Rightarrow y=\iota_{2} * \\ y=\iota_{2} * & \Rightarrow \text { true }\end{cases}
$$

To illustrate behaviour refinement, consider the lossy buffer LBuff introduced in section 2, and a deterministic buffered channel Buff specified as a coalgebra $M^{*} \longrightarrow\left(M^{*} \times(\mathbf{1}+M)\right)^{M+\mathbf{1}}$ with nil as the initial state, and dynamics given by

$$
\begin{aligned}
a_{\text {Buff }}\langle u, \text { put } m\rangle & =\left\langle m: u, \iota_{1} *\right\rangle \\
a_{\text {Buff }}\langle u, \text { pick }\rangle & =\left\langle\text { tail } u, \iota_{2}(\text { head } u)\right\rangle
\end{aligned}
$$

To establish LBuff $\unlhd$ Buff it is required first to embed the latter into the space of non-deterministic systems. This is achieved by a (natural) transformation from $(\mathrm{Id} \times O)^{I}$ to $\mathcal{P}(\mathrm{Id} \times O)^{I}$ canonically extending function $\operatorname{sing} x=\{x\}$ which is a monad morphism from the identity to the powerset monads - the behaviour models underlying Buff and LBuff, respectively. Then, it is immediate to verify that the identity function on state space $M^{*}$ is a forward morphism, with respect to the first preorder given above, i.e.,

$$
\text { (id } \times O) \cdot a_{\text {Buff }} \subseteq a_{\text {LBuff }}
$$

Another behaviour refinements of LBuff would arise by choosing different strategies for delivering elements from the buffer. Here are some possibilities, each of them is witnessed by a forward morphism:

- the queuing strategy, leading to the specification Buff as above;

- the stack strategy (LIFO deliver);

- the priority strategy (in which elements carry some probability information);

- the lift strategy (a linear order on the elements is served in alternating increasing and decreasing order).

In the priority strategy, for example, elements are labelled with a 'show-up' probability, introducing an elementary form of probabilistic nondeterminism. As detailed in [3], the corresponding behaviour monad is generated by a monoid $M=\langle[0,1], \min , \times\rangle$ with the additional requirement that for each $m \in M$, $\sum\left(\mathcal{P} \pi_{2}\right) m=1$. 'Probabilistic' components can be embedded into the space of 'plain nondeterministic' ones where behaviour refinement, wrt $\subseteq_{\mathrm{T}}$, is discussed. 


\section{Simulations}

In this section we prove that behaviour refinement, as characterized above, can be established by a simulation relation $R \subseteq U_{p} \times U_{q}$ on the state spaces of the 'concrete' $(p)$ and the 'abstract' $(q)$ components. Again, the notion of a simulation depends on the adopted refinement preorder $\leq$. To proceed in a generic way, we adopt an equally generic definition of simulation due to Jacobs and Hughes in $[9]$ :

Definition 4 Given a Set endofunctor $\mathrm{T}$ and a refinement preorder $\leq$, a lax relation lifting is an operation $\operatorname{Rel}_{\leq}(\mathrm{T})$ mapping relation $R$ to $\leq \circ \operatorname{Rel}(\mathrm{T})(R) \circ \leq$, where $\operatorname{Rel}(\mathrm{T})(R)$ is the lifting of $R$ to $\mathrm{T}$ (defined, as usual, as the $\mathrm{T}$-image of inclusion $\left\langle r_{1}, r_{2}\right\rangle: R \longrightarrow U \times V$, i.e., $\left.\left\langle\mathrm{T} r_{1}, \mathrm{~T} r_{2}\right\rangle: \mathrm{T} R \longrightarrow \mathrm{T} U \times \mathrm{T} V\right)$.

Given $\mathrm{T}$-coalgebras $\alpha$ and $\beta$, a simulation is a $\operatorname{Re}_{\leq}(\mathrm{T})$-coalgebra over $\alpha$ and $\beta$, i.e., a relation $R$ such that, for all $u \in U, v \in V,\langle u, v\rangle \in R \Rightarrow\langle\alpha u, \beta v\rangle \in$ $\operatorname{Rel}_{\leq}(\mathrm{T})(R)$.

In order to prove that simulations are a sound proof technique to establish behaviour refinement we consider separately functional and non functional simulations. In any case, however, simulations are assumed to be left total relations ${ }^{6}$ as we do not consider partial refinements.

Lemma 4. Let $p$ and $q$ be $T$-components over state spaces $U$ and $V$, respectively. For a given refinement preorder $\leq$, if there exists a simulation $R \subseteq U \times V$ which is both functional and left total, then $p$ is a (forward) refinement of $q$.

Proof. By assumption, simulation $R$ is the graph of a function. Now, define a forward morphism $h: U \rightarrow V$ as $h u=v$ iff $\langle u, v\rangle \in R$. Because $R$ is a simulation, for every pair $\langle u, v\rangle \in R$, there should exist $x \in \mathrm{T} U, y \in \mathrm{T} V$, such that $\alpha u \leq_{\mathrm{T} U} x, y \leq_{\mathrm{T} V} \beta v$, and $\langle x, y\rangle \in \operatorname{Rel}(\mathrm{T})(R)$, i.e., $y=\mathrm{T} h(x)$. By (10) and $\alpha u \leq_{\mathrm{T} U} x$ we get $\mathrm{T} h(\alpha u) \leq_{\mathrm{T} V} \mathrm{~T} h(x)$, and thus $\mathrm{T} h(\alpha u) \leq_{\mathrm{T} V} \beta v$. Since $R$ is left total, $h$ is defined for all $u \in U$, making the following diagram to commute:

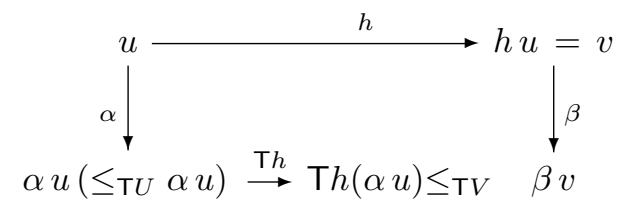

Consider, now, the non-functional case (e.g. whenever two bisimilar but not equal abstract states are represented by a single concrete state). To prove soundness in this case, the state space of the 'concrete' component $p$ is artificially inflated with an auxiliary state space such that a forward morphism can be found.

\footnotetext{
${ }^{6}$ A relation $R \subseteq U \times V$ is functional if every $u \in U$ is related to at most one $v \in V$
} and left total if for all $u \in U$, there exists some $v \in V$ such that $\langle u, v\rangle \in R$. 
Definition 5 Given a coalgebra $(U, \alpha: U \rightarrow \mathrm{T} U)$ and a set $W$, define the extension of $\alpha$ to $W$ as any coalgebra $\widehat{\alpha}$ over $\widehat{U}=U \times W$ such that $\mathrm{T} \pi_{1} \circ \widehat{\alpha}=\alpha \circ \pi_{1}$.

Clearly this auxiliary state space does not interfere with the behaviour of $\alpha: \pi_{1}$ being a coalgebra morphism, the two coalgebras are bisimilar.

Given components $p$ and $q$ and a non-functional simulation $R$ an auxiliary coalgebra $\widehat{p}$ can be defined taking $R$ as the state space (which, because $R$ is left total, is just an extension of $p$ in the sense of the definition above) and the rule $\left(u^{\prime}, v^{\prime}\right) \in_{\mathrm{T}} \widehat{\alpha}(u, v)$ iff $u^{\prime} \in_{\mathrm{T}} a_{p} u \wedge v^{\prime} \in_{\mathrm{T}} a_{q} v$ as its dynamics. With this construction we prove that

Lemma 5. (Soundness) To prove $q \unlhd p$ it is sufficient to exhibit a left total simulation $R$ relating components $p$ and $q$.

Proof. If $R$ is functional the result follows from lemma 4. Otherwise construct $\widehat{p}$ as above: clearly $p$ is bisimilar to $\widehat{p}$ and the graph of projection $\pi_{2}$ from its state space to $V$ defines a simulation between $\widehat{p}$ and $q$. By definition, $p \sim \widehat{p}$ and the existence of a (seed-preserving) forward morphism from $\widehat{p}$ to $q$ entails $q \unlhd p$.

Finally notice that, although $\unlhd$ is transitive, it is not always the case that simulations are closed under (relational) composition. This would be a consequence of $\operatorname{Rel}_{\leq}(\mathrm{T})$ preserving composition, but, in general, only the following weaker result holds:

Lemma 6. Any refinement preorder $\leq$ verifies

$$
\operatorname{Re}_{\leq}(\mathrm{T})(R \circ S) \subseteq \operatorname{Re}_{\leq}(\mathrm{T})(R) \circ \operatorname{Rel}_{\leq}(\mathrm{T})(S) \text { and } \quad=_{\mathrm{T} U} \subseteq \operatorname{Re}_{\leq}(\mathrm{T})\left(=_{U}\right)
$$

Proof. For the first statement note that $\langle u, w\rangle \in \operatorname{Re}_{\leq}(\mathrm{T})(R \circ S)$ equivales

$$
\begin{aligned}
& \exists u^{\prime}, w^{\prime} .\left(u \leq u^{\prime} \wedge\left\langle u^{\prime}, w^{\prime}\right\rangle \in \operatorname{Rel}(\mathbf{T})(R \circ S) \wedge w^{\prime} \leq w\right) \\
& \{\text { because } \operatorname{Rel}(\mathbf{T})(R \circ S)=\operatorname{Rel}(\mathbf{T})(R) \circ \operatorname{Rel}(\mathbf{T})(S)\} \\
\Leftrightarrow & \exists u^{\prime}, w^{\prime} .\left(u \leq u^{\prime} \wedge\left(\exists v^{\prime} .\left(\left\langle u^{\prime}, v^{\prime}\right\rangle \in \operatorname{Rel}(\mathbf{T})(R) \wedge\left\langle v^{\prime}, w^{\prime}\right\rangle \in \operatorname{Rel}(\mathbf{T})(S)\right)\right) \wedge w^{\prime} \leq w\right) \\
\Leftrightarrow & \exists u^{\prime}, w^{\prime}, v^{\prime} .\left(u \leq u^{\prime} \wedge\left\langle u^{\prime}, v^{\prime}\right\rangle \in \operatorname{Rel}(\mathbf{T})(R) \wedge\left\langle v^{\prime}, w^{\prime}\right\rangle \in \operatorname{Rel}(\mathbf{T})(S) \wedge w^{\prime} \leq w\right) \\
& \left\{\text { introducing } v=v^{\prime}\right\} \\
\Rightarrow & \exists u^{\prime}, w^{\prime}, v, v^{\prime} .\left(u \leq u^{\prime} \wedge\left\langle u^{\prime}, v^{\prime}\right\rangle \in \operatorname{Rel}(\mathbf{T})(R) \wedge v^{\prime} \leq v\right) \wedge \\
& \left(v \leq v^{\prime} \wedge\left\langle v^{\prime}, w^{\prime}\right\rangle \in \operatorname{Rel}(\mathbf{T})(S) \wedge w^{\prime} \leq w\right) \\
\Rightarrow & \exists v \cdot\langle u, v\rangle \in \operatorname{Rel}_{\leq}(\mathrm{T})(R) \wedge(v, w) \in \operatorname{Rel}_{\leq}(\mathrm{T})(S) \\
\Leftrightarrow & \langle u, w\rangle \in \operatorname{Rel}_{\leq}(\mathrm{T})(R) \circ \operatorname{Rel}_{\leq}(\mathrm{T})(S)
\end{aligned}
$$

Then consider

$$
\begin{aligned}
=_{\mathrm{T} U} & \subseteq \leq_{\mathrm{T} U} \\
& =\leq_{\mathrm{T} U} \circ=_{\mathrm{T} U} \circ \leq_{\mathrm{T} U}=\leq_{\mathrm{T} U} \circ \operatorname{Rel}(\mathrm{T})\left(=_{U}\right) \circ \leq_{\mathrm{T} U}=\operatorname{Rel}_{\leq}(\mathrm{T})\left(=_{U}\right)
\end{aligned}
$$




\section{Discussion and Future Work}

In this paper, two levels of refinement for (state-based) components have been introduced. In particular, the notion of behavioural refinement parametric on a model of behaviour captured by a strong monad B is, to the best of our knowledge, new. It is generic enough to capture a number of situations, depending on both B and the refinement preorder adopted. Nondeterminism reduction is just one possibility among many others. Also note that Poll's notion of behavioural subtyping in [13], at the model level, emerges as a particular instantiation.

As mentioned in the introduction, the main motivation underlying this work is the development of inequational laws in the context of the component calculus proposed in [3]. Even though there is not enough space in this paper to introduce the derived laws, let us take a brief glimpse. As a first example consider equation

$$
\left\ulcorner!_{I}\right\urcorner \sim p ;\left\ulcorner!_{O}\right\urcorner
$$

which does not hold for non trivial behaviour models. In fact the $\mathrm{Cp}$ lifting of the final arrow (as the lifting of any other function) cannot fail, whereas the the right hand side may fail (whenever $p$ does). Function ! : $U_{p} \times \mathbf{1} \longrightarrow \mathbf{1}$ is, however, a forward morphism, with respect to $\subseteq_{\mathrm{T}}^{F}$ for partial components, or to both $\subseteq_{\mathrm{T}}$ and $\subseteq_{\mathrm{T}}^{\mathrm{E}}$ for non deterministic ones. For this last case, note that $\bar{a}_{\left\ulcorner!_{O}\right\urcorner \cdot !}=\lambda i \in I .\{*\}$, whereas $\mathrm{B}(! \times \mathrm{id})^{I} \cdot \bar{a}_{p ;\left\ulcorner!_{O}\right\urcorner}\langle u, *\rangle$ equals

$$
\lambda i \in I . \begin{cases}\{*\} & \text { iff }\left(\bar{a}_{p} u\right)(i) \neq \emptyset \\ \emptyset & \text { iff }\left(\bar{a}_{p} u\right)(i)=\emptyset\end{cases}
$$

Therefore, $\left\ulcorner!_{I}\right\urcorner \unlhd p ;\left\ulcorner!_{O}\right\urcorner$. Similarly, the cancellation law for parallel composition $\bigotimes$, which involves a split-like construction for components (which, differently from the split of functions [4], is not an universal arrow), is, in general, a refinement result:

$$
p \unlhd\langle p, q\rangle ;\left\ulcorner\pi_{1}\right\urcorner
$$

witnessed by projection $\pi_{1}: U_{p} \times U_{q} \times 1 \rightarrow U_{p}$ as a forward morphism. Yet another example is given by the (pseudo) naturality of $\ulcorner\Delta\urcorner$, where $\Delta$ is the diagonal function, which could be written as

$$
\ulcorner\Delta\urcorner ;(p \otimes p) \unlhd p ;\ulcorner\triangle\urcorner
$$

Finally, monotonicity of $\unlhd$ with respect to both pipeline composition and the tensor products can be proved by defining a simulation in terms of the argument simulations: if $q \unlhd p$ and $t \unlhd r$ are witnessed by $R_{1}$ and $R_{2}$, respectively, refinement $q \square t \unlhd p \square r$, with $\square$ standing for ;, $\otimes, \boxplus$ or $⿴ 囗 ⿱ 一 一)$ is witnessed by simulation $R=$ $\left\{\left(\left(u_{p}, u_{r}\right),\left(u_{q}, u_{t}\right)\right) \mid\left(u_{p}, u_{q}\right) \in R_{1} \wedge\left(u_{r}, u_{t}\right) \in R_{2}\right\}$.

Currently we are working on the full development of the refinement calculus and, in particular, in its application to the proof of consistency between static and dynamic diagrams in UML in the context of [12]. Whether this approach scales up to be useful in the classification and transformation of software architectures [1] remains a research question. Further comparison with refinement 
theories in both process algebra (as in, e.g., [5]) and state-based systems (for example in [6]) is also in order.

Acknowledgements. This piece of research was carried on in the context of the PURE Project (Program Understanding and Re-engineering) supported by FCT (the Portuguese Foundation for Science and Technology) under contract POSI/ICHS/44304/2002. The work of Sun Meng was further supported by the National Natural Science Foundation of China under grant no. 60273001.

\section{References}

1. R. Allen and D. Garlan. A formal basis for architectural connection. ACM TOSEM, 6(3):213-249, 1997.

2. L. S. Barbosa. Towards a Calculus of State-based Software Components. Journal of Universal Computer Science, 9(8):891-909, August 2003.

3. L. S. Barbosa and J. N. Oliveira. State-based components made generic. In H. P. Gumm, editor, CMCS'03, Elect. Notes in Theor. Comp. Sci., volume 82.1, 2003.

4. R. Bird and O. Moor. The Algebra of Programming. Series in Computer Science. Prentice-Hall International, 1997.

5. P. Degano, R. Gorrieri, and G. Rosolini. A categorical view of process refinement. In J. de Bakker, G. Rozenberg, and J. Rutten, editors, Proc. REX Workshop on Semantics, pages 138-154. Springer Lect. Notes Comp. Sci. (666), 1992.

6. J. Derrick and E. Boiten. Calculating upward and downward simulations of statebased specifications. Information and Software Technology, 41:917-923, July 1999.

7. M. Fokkinga and R. Eshuis. Comparing refinements for failure and bisimulation semantics. Technical report, Faculty of Computing Science, Enschede, 2000.

8. C. A. R. Hoare. Proof of correctness of data representations. Acta Informatica, $1: 271-281,1972$.

9. B. Jacobs and J. Hughes. Simulations in coalgebra. In H. P. Gumm, editor, CMCS'03, Elect. Notes in Theor. Comp. Sci., volume 82.1, Warsaw, April 2003.

10. C. B. Jones. Systematic Software Development Using VDM. Series in Computer Science. Prentice-Hall International, 1986.

11. B. Liskov. Data abstraction and hierarchy. SIGPLAN Notices, 23(3), 1988.

12. S. Meng and B. Aichernig. Towards a Coalgebraic Semantics of UML: Class Diagrams and Use Cases. Technical Report 272, UNU/IIST, January 2003.

13. E. Poll. A coalgebraic semantics of subtyping. Theorectical Informatica and Apllications, 35(1):61-82, 2001.

14. J. Rutten. Universal coalgebra: A theory of systems. Theor. Comp. Sci., 249(1):380, 2000. (Revised version of CWI Techn. Rep. CS-R9652, 1996).

15. C. Szyperski. Component Software, Beyond Object-Oriented Programming. Addison-Wesley, 1998.

16. P. Wadler and K. Weihe. Component-based programming under different paradigms. Technical report, Dagstuhl Seminar 99081, February 1999.

17. J. Woodcock and J. Davies. Using Z: Specification, Refinement and Proof. Prentice-Hall International, 1996. 